\title{
Science rides the online wave
}

A lthough scientists today rely on the World Wide Web to help them with their research, mainly using it to search for information, newer capabilities such as blogging, tagging, and social networking are only just beginning to be exploited by scientists.

Over the past few years, the changes that have taken place in the ways that the World Wide Web is used are epitomized by the dramatic increase in the number of social-networking websites (communities of people who share information), wikis (collections of web pages that can be added to and modified by anyone who has access to them), and blogs (websites with regular entries on a particular subject or by a particular individual). Although there have been no technical upgrades to facilitate this new use of the World Wide Web to share and edit information as well as to collaborate, the new capabilities have been termed Web 2.0.

The capabilities of Web 2.0 are behind the new, clinically relevant social-networking website www.MalariaEngage.org, which aims to open a channel between the global internet community and African scientists who are working to eradicate one of the biggest health problems on their continent - malaria. The website, which was launched in time for World Malaria Day on April 25 , is a collaboration between researchers at the University Health Network and the University of Toronto-based McLaughlinRotman Centre (MRC) for Global Health; Impactanation, an organization dedicated to increasing awareness and inspiring entrepreneurial action in young people around the themes of environment, health, and social justice; and the National Institute for Medical Research (NIMR) in Tanzania.

The MalariaEngage website currently highlights seven research projects being conducted by scientists at the NIMR in Tanzania. Each addresses a different aspect of research into malaria treatment and/or prevention, ranging from how to improve the use of insecticide-treated bed nets to assessing whether judicious planting of vegetation with mosquito-repellent properties might provide benefit. Peter A. Singer, one of the MRC researchers responsible for the website, was keen to stress to the JCI that the highlighted projects were chosen by the NIMR in Tanzania after a competitive internal peer review of proposals. Singer believes ensuring that scientific accountability for the projects remains with the NIMR in Tanzania is key to the aims of the MalariaEngage project - supporting African scientists.

Although the use of Web 2.0 tools enables individuals visiting the site to make donations directly to an individual research project, the website is not purely a way to seek investment. Indeed, Singer says the main idea behind the website is to engage with a group of people he feels was falling through the cracks in the global effort to control malaria - African

scientists looking

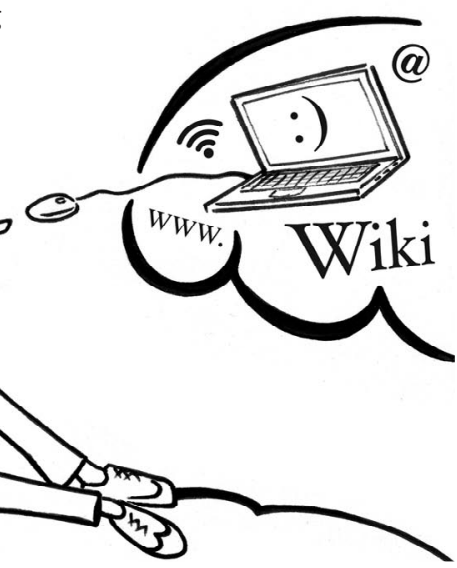

Another way some scientists are exploiting Web 2.0 tools is sharing detailed information about their protocols and results (positive and negative) online. Many of these sites are wikis that users say promote the sharing of information and collaboration between users. One widely used wiki is www.OpenWetWare.org, a website created in 2005 by graduate students in two laboratories at the Massachusetts Institute of Technology as a way to keep the laboratory websites up to date. However, it soon became a place to share information about protocols, and it has now grown to a dynamic resource providing insight into many things including protocols, college courses, conferences, and careers.

Users of OpenWetWare, such as Maureen Hoatlin, an assistant professor at Oregon Health \& Science University, say they love the interaction among users and believe that it has a tremendous power to move science forward (2). But many other scientists remain skeptical about the degree of openness provided by wikis, fearing they will get scooped and lose the credit for a new discovery. Even supporters of these sites acknowledge that there are issues created by the openness of wikis that need to be addressed. For example, JeanClaude Bradley, an associate to address questions not otherwise being asked. Each research project has several areas powered by Web 2.0 tools to facilitate this, including a discussion forum where interested parties can interact with each other and the researchers and a site where advances in the research will be posted.

One criticism of the website, raised by Anna Wang, public affairs officer with the Medicines for Malaria Venture in Geneva, is that it is hard for those who are not scientists or clinicians to evaluate the scientific merit of the projects (1). However, Singer counters that this should not be a problem because individuals can use the Web 2.0 capabilities of the website to ask the scientists directly what they are doing and why. professor in the Department of Chemistry at Drexel University who created his own laboratory wiki (www.usefulchem.wikispaces.com), says that until issues such as whether a wiki posting constitutes proof of priority for filing a patent is sorted out, "the typical legal advice is: do not disclose your ideas before you file” (2).

\section{Karen Honey}

1. Hopkin, M. 2008 April 22. Social networking site aims to help fight malaria. Nature News. http://www.nature.com/news/2008/080422/full/ news.2008.768.html.

2. Waldrop, M.M. 2008 April 21. Science 2.0 - Is open access science the future? Scientific American Magazine. http://www.sciam.com/article.cfm?id=science2-point-0. 